\title{
Can the analysis of BH3-only protein knockout mice clarify the issue of 'direct versus indirect' activation of Bax and Bak?
}

\author{
A Villunger ${ }^{\star, 1}$, V Labi $^{1}$, P Bouillet ${ }^{2,3}$, J Adams ${ }^{2,3}$ and A Strasser ${ }^{\star, 2,3}$ \\ Cell Death and Differentiation (2011) 18, 1545-1546; doi:10.1038/cdd.2011.100
}

A recent study by Ren et al. ${ }^{1}$ contributes to the ongoing debate about how interactions between factions of the $\mathrm{Bcl}-2$ protein family provoke apoptosis, but the data presented do not, in our view, support the overall conclusion that 'Bid, Bim and Puma are essential for activation of the Bax- and Bak-dependent cell death program'.

It is generally accepted that, in response to diverse cellular stresses, the $\mathrm{Bcl}-2$ distant cousins termed 'BH3-only proteins', for example, Bim, Bid or Puma, initiate the apoptotic process and that the pivotal step (mitochondrial outer membrane permeabilization) requires the proapoptotic $\mathrm{Bcl}-2$ family members Bak or Bax, ${ }^{2}$ but how the BH3-only proteins provoke activation of Bax and Bak remains controversial. The 'direct activation' model ${ }^{3}$ posits that Bim, Bid and possibly Puma serve as direct 'activators'. In healthy cells, prosurvival $\mathrm{Bcl}-2$ proteins sequester them, but cytotoxic stimuli upregulate or activate 'sensitizer BH3-only proteins' (Bad, Bik, Hrk, Noxa and Bmf) whose binding to the prosurvival $\mathrm{Bcl}-2$ proteins liberates the 'activators' to transiently engage and activate Bax/Bak. Conversely, the 'indirect model' ${ }^{4}$ postulates that in healthy cells a small proportion of Bax and Bak is primed to elicit cell death but sequestered by prosurvival $\mathrm{Bcl}-2$ proteins, and that $\mathrm{BH} 3-o n l y$ proteins must engage all prosurvival proteins in a given cell to unleash Bax/Bak for death duty. This can either be achieved by Bim, Puma or Bid, which can bind all their prosurvival relatives, or by combinations of $\mathrm{BH} 3-$ only proteins that bind complementary subsets (e.g., Bad, binding $\mathrm{Bcl}-2$, Bcl- $\mathrm{x}_{\mathrm{L}}$ and $\mathrm{Bcl}-\mathrm{w}$, plus Noxa, binding $\mathrm{Mcl}-1$ and $\left.\mathrm{A} 1^{4,5}\right)$. Although biochemical studies have provided conflicting results, the indirect activation model was supported by the observation that Bax/Bak double-deficient (DKO) mice ${ }^{2}$ have much more severe developmental and apoptotic defects than mice lacking Bim and Bid, ${ }^{4}$ the two most widely accepted 'direct activators' within the Bcl-2 family.

Ren et al. ${ }^{1}$ generated Bim/Bid/Puma triple-deficient (TKO) mice to resolve whether Puma also functions as a 'direct activator' and to clarify the mechanisms of Bax/Bak activation. They report that triple deficiency for Bim, Bid and Puma mirrors Bax/Bak double deficiency and argue that this provides proof for the 'direct activation' model. This is, however, incorrect. First, there are substantial differences in phenotype between Bim/Bid/Puma TKO ${ }^{1}$ and Bax/Bak DKO mice. $^{2}$ Although Bax/Bak DKO mice die perinatally with severe brain abnormalities, no such profound neurological defects and associated perinatal lethality were reported for the Bim/Bid/Puma TKO mice. Furthermore, although some interdigital webbing persisted in Bim/Bid/Puma TKO mice, ${ }^{1}$ it appears less extensive than in Bax/Bak DKO mice ${ }^{2}$ or in $\mathrm{Bim}^{-1-} \mathrm{Bmf}^{-1-}$ mice $^{6}$ (AV, PB and VL, unpublished), in which webs persist despite the presence of both Bid and Puma. Moreover, the defect in vaginal development in Bim/Bid/Puma TKO mice shows incomplete penetrance, ${ }^{1}$ but occurs in $100 \%$ of Bax/Bak DKO mice. ${ }^{2}$ Thus, in a significant portion of Bim/Bid/Puma TKO mice, the physiological cell death driven by Bax and/or Bak continues to some extent in multiple tissues.

The reported in vitro cell survival assays also fail to unambiguously demonstrate that all induction of apoptosis requires Bid, Bim or Puma. A proportion of the TKO lymphoid cells still died in response to DNA damage or glucocorticoids, ${ }^{1}$ whereas Bax/Bak DKO cells are fully refractory. ${ }^{2}$ This difference may indicate that these death stimuli activate additional (i.e., non 'direct activator') BH3-only proteins that collectively can neutralize the prosurvival $\mathrm{Bcl}-2$ proteins in these cells, thereby leading to Bax/Bak activation, consistent with the 'indirect model'. Moreover, the role of Bid in the lymphocyte death probably is negligible: although this study failed to provide data on survival of Bim/Puma DKO lymphocytes, previous studies ${ }^{7,8}$ have shown that their combined loss renders multiple hematopoietic cell types as resistant to the apoptotic stimuli studied as reported for the Bim/Bid/Puma TKO cells. ${ }^{1}$

Thus, the phenotype of the TKO mice is less profound than that of Bax/Bak DKO animals and does not prove the direct activation model. As the TKO mice lack the three $\mathrm{BH}$ only proteins that can neutralize all the prosurvival family members, ${ }^{5}$ the observed apoptotic deficiencies are also

\footnotetext{
${ }^{1}$ Division of Developmental Immunology, Biocenter, Innsbruck Medical University, Innsbruck, Austria; ${ }^{2}$ Molecular Genetics of Cancer Division, Walter and Eliza Hall Institute of Medical Research, Melbourne, Victoria, Australia and ${ }^{3}$ Department of Medical Biology, University of Melbourne, Melbourne, Victoria, Australia ${ }^{*}$ Corresponding authors: A Villunger, Division of Developmental Immunology, Biocenter, Innsbruck Medical University, A-6020 Innsbruck, Austria. Tel: + 435129003 70380; Fax: + 435129003 73960; E-mail: andreas.villunger@ i-med.ac.at or A Strasser, Molecular Genetics of Cancer Division, Walter and Eliza Hall Institute of Medical Research, 1G Royal Parade, Melbourne, 3050 Victoria, Australia. Tel: +61 39345 2624; Fax: + 6139347 0852; E-mail: strasser@wehi.edu.au
} 
compatible with the 'indirect model'. Nevertheless, increasing in vitro findings, for example, ${ }^{9,10}$ suggest that certain $\mathrm{BH} 3$ domains can directly activate $\mathrm{Bax}$, and a recent in vivo study using gene-targeted mice in which the $\mathrm{BH} 3$ region of Bim has been subtly altered argues that aspects of both models may well hold. ${ }^{11}$ Most of the seemingly conflicting published results can be reconciled if Bax and Bak can be activated in multiple ways: in some circumstances by Bid, Bim or Puma, but also, albeit perhaps less efficiently, by certain other BH3-only proteins, ${ }^{12}$ or by mechanisms independent of BH3-only proteins, ${ }^{3}$ such as by Bax phosphorylation, heatinduced conformational change, or spontaneous activation after the neutralization or degradation of the restraining prosurvival $\mathrm{Bcl}-2$ proteins, as seen in platelets. As small molecules that target prosurvival $\mathrm{Bcl}-2$ proteins are showing great clinical promise, it will be essential to understand these mechanisms to achieve optimal killing of tumor cells.

1. Ren D et al. Science (New York, NY) 2010; 330: 1390.

2. Lindsten T et al. Mol Cell 2000; 6: 1389 .

3. Chipuk JE, Green DR. Trends Cell Biol 2008; 18: 157

4. Willis SN et al. Science (New York, NY) 2007; 315: 856

5. Chen L et al. Mol Cell 2005; 17: 393.

6. Hubner A et al. Mol Cell Biol 2010; 30: 98.

7. Erlacher M et al. J Exp Med 2006; 203: 2939

8. Karlberg $\mathrm{M}$ et al. Cell Death Dis 2010; 1 : e43.

9. Gavathiotis E et al. Nature 2008; 455: 1076.

10. Lovell JF et al. Cell 2008; 135: 1074.

11. Merino D et al. J Cell Biol 2009; 186: 355

12. $\mathrm{Du} \mathrm{H}$ et al. J Biol Chem 2010; 286: 491-501. 